\title{
Effects of volatile compounds in apple juices on fungal growth and patulin production of Penicillium expansum
}

\author{
Tomoyasu TAGUCHI, ${ }^{1, *}$, Atsushi IshIHARA ${ }^{2}$, and Hiromitsu NAKAJIMA ${ }^{2}$ \\ ${ }^{1}$ Environmental Hygiene Chemistry Group, Quality Assessment Center 1, Quality Food Research Laboratories, Division of \\ Research and Development, Meiji Co., Ltd., 540 Naruda, Odawara, Kanagawa 250-0862, Japan \\ ${ }^{2}$ Department of Agricultural Chemistry, Faculty of Agriculture, Tottori University, Koyama, Tottori 680-8553, Japan
}

Key words : 2-methylbutanoic acid; patulin; patulin production; Penicillium expansum; volatile compounds

(Received November 8, 2013; Accepted December 7, 2013)

\begin{abstract}
Penicillium expansum was cultured in apple juice media prepared from six different commercial apple juices. The patulin production was profoundly affected by the differences in apple juices, whereas fungal growth was generally not. The maximum concentration of patulin was 7.3 -fold of that in the media containing minimum concentration of patulin. The six apple juices were concentrated by evaporation and reconstructed to the original volumes by adding Milli-Q water. P. expansum was cultured in the media prepared from reconstructed apple juices, and the patulin concentration and fungal growth were determined. Evaporation of apple juice tended to decrease patulin production and to increase fungal growth, suggesting that the volatile compounds promote patulin production and inhibit fungal growth. The volatile compounds in the apple juice were then analyzed by GC-MS. The 13 compounds of which concentrations were largely decreased by evaporation were selected for evaluation of their stimulation of patulin production. Seven out of the 13 compounds, 2-methylpropyl acetate, ethyl butyrate, ethyl 2-methylbutanoate, 3-methyl-1-butanol, hexyl acetate, 1-hexanol, and 2-methylbutanoic acid, increased the patulin production of $P$. expansum concentration-dependently; 2-methylbutanoic acid and its ethyl ester were highly effective. Our results indicated that the composition of volatile compounds in apple juice media largely affects the patulin production and the growth of $P$. expansum.
\end{abstract}

\section{Introduction}

Patulin is one of the mycotoxins, first isolated in 1940 from a culture of Penicillium patulum (also called P. griseofulvum $)^{1)}$, and is a major contaminant for apples and pears ${ }^{2)}$. Patulin shows immunotoxic and neurotoxic effects in animal experiments, ${ }^{3,4)}$, and is regulated in many countries, including the U.S. ${ }^{5}$, the European Union $^{6)}$, and Japan ${ }^{7}$. These countries have adopted $50 \mu \mathrm{g} / \mathrm{kg}$ as a maximum residue limit in apple juice and apple-related foods. Many species of Penicillium and Aspergillus such as P. patulum, P. melinii, P. claviforme, A. clavatus, A. giganteus, and A. terreus produce patulin, and the most common species that causes patulin contamination in apples is $P$. expansum ${ }^{2)}$.

\footnotetext{
Corresponding Author

*Environmental Hygiene Chemistry Group, Quality Assessment Center 1, Quality Food Research Laboratories, Division of Research and Development, Meiji Co., Ltd., 540 Naruda, Odawara, Kanagawa 250-0862 Japan. Tel: +81-465-37-3667. Fax: +81-465-37-3640. E-mail: tomoyasu.taguchi@meiji.com
}

A full color PDF reprint of this article is available at the journal WEB site. 
Patulin contamination in foods results in great economic loss ${ }^{8)}$, and thus food industries are eager to develop a technique to prevent patulin contamination in apple juice. Until now, many investigations for prevention the growth of $P$. expansum and patulin contamination have been performed. Patulin production in apples usually occurs after harvesting. Appropriate quality control of postharvest apples, such as adequate handling and storage conditions, is very important to prevent patulin contamination ${ }^{9-13)}$. The Codex Alimentarius Commission (CAC) has been preparing guidelines to reduce patulin in apples. Chemical agents such as potassium sorbate and sodium propionate inhibit $P$. expansum growth and patulin production, ${ }^{94}$, and UV irradiation reduces patulin contents in apple cider $^{15}$. However, patulin contamination of apples is still not adequately controlled.

Some natural chemicals contributing to plant aroma have antibacterial and antifungal activities ${ }^{16-22)}$. These chemicals can reduce the risk of contamination of agricultural products and foods, and the health hazards presented by microorganisms. In particular, cinnamaldehyde, benzaldehyde, and aliphatic aldehydes were found to have potent inhibitory effects against microorganisms. Among these, (Z)-3-hexenal, 2,4-hexadienal, and (E)-2-undecenal showed relatively strong antibacterial and antifungal effects, and those compounds prevented the production of toxic secondary metabolites. Gaseous (E)-2-hexenal was effective for preventing $P$. expansum contamination in pears ${ }^{23}{ }^{24)}$. In our previous study, the effects of 16 aliphatic aldehydes with 3-10 carbons on the growth and patulin production of $P$. expansum were examined, and we found that 2-propenal, $(E)$-2-butenal, $(E)$-2-pentenal, and (E)-2-hexenal added to apple juice media inhibited the germination of $P$. expansum spores and fungal growth ${ }^{25)}$.

On the other hand, gaseous (E)-2-heptanal, $(E)$-2-octenal, and $(E)$-2-nonenal have been reported to stimulate aflatoxin production by A. flavus inoculated into corn, but to suppress it in cotton seed and peanuts ${ }^{26)}$. In our study the aliphatic aldehydes with 8-10 carbons, octanal, (E)-2-octenal, nonanal, (E)-2-nonenal, decanal, and (E)-2-decenal added to the apple juice medium significantly enhanced the patulin production by $P$. expansum without affecting fungal growth ${ }^{25)}$. These findings suggest that volatile compounds in apples and apple juice affect the patulin production by P. expansum growing in apples and apple juice.

Karlshøj et al. (2007) investigated the composition of volatile compounds by electric nose analysis to clarify the relationship between $P$. expansum infection and patulin spoilage in apples ${ }^{27)}$. To the best of our knowledge, however, there is no report on the effects of individual volatile compounds in apples on the patulin production by $P$. expansum. Here we describe the effect of volatile compounds in apple juice on the patulin production by $P$. expansum, and we discuss our finding that some of the compounds, such as 2-methylbutanoic acid and its ethyl ester, increased the patulin contents in apple juice culture concentrationdependently.

\section{Materials and Methods}

Apple juices Clear apple juices A-F made by six different domestic companies were purchased from grocery stores and stored at $4^{\circ} \mathrm{C}$ in dark until experiment. Reconstructed apple juices were prepared as follows: apple juices were concentrated by a rotary vacuum evaporator equipped with a vacuum controller NVC-1100 (Tokyo Rikakikai Co., Tokyo, Japan) at $45^{\circ} \mathrm{C}$. The respective concentration rates were 7.5, 7.0, $6.9,7.1,7.6,8.0$ for apple juices A-F. The concentrates were filled up to their original volumes by adding Milli-Q water. Reconstructed apple juices were also stored at $4^{\circ} \mathrm{C}$ in dark until experiment. 
Chemicals Patulin standard was purchased from Wako Pure Chemical Industries (Osaka, Japan). The 13 volatile compounds were purchased from Wako or from Tokyo Chemical Industry Co. (Tokyo, Japan). Ethanol $(\mathrm{EtOH})$, methanol $(\mathrm{MeOH})$, hydrochloric acid $(\mathrm{HCl})$, and sodium chloride $(\mathrm{NaCl})$ were of analytical grade, and acetonitrile (MeCN) was of high-performance liquid chromatography (HPLC) grade.

Fungal strain and spore preparation Penicillium expansum ATCC28876 was purchased from the American Type Culture Collection (Rockville, MD, USA). P. expansum spore suspension was prepared and preserved as described ${ }^{25)}$.

Patulin production by $\mathbf{P}$. expansum in six media made from different commercial apple juices and the effect of evaporation of apple juices on patulin production Heated apple juice media were prepared from apple juices A-F by sterilization at $121^{\circ} \mathrm{C}$ for $10 \mathrm{~min}$. These media are referred to as AJMs (apple juices media) A-F in this study. Reconstructed apple juice media were also prepared from reconstructed apple juices A-F by sterilization, and are referred to as RAJMs (reconstructed apple juices media) A-F.

P. expansum spores were inocultaed to apple juice media $(10 \mathrm{~mL})$ in $50-\mathrm{mL}$ cell culture flasks (Becton Dickinson, Franklin Lakes, NJ, USA) with the final spore concentration of $1 \times 10^{4}$ spores/mL and cultured statically at $25^{\circ} \mathrm{C}$ for $7 \mathrm{~d}$. The dry weight of mycelia was measured as an indicator of fungal growth, and the patulin concentration in the culture medium was determined. The differences in the patulin concentrations and the dry weights of mycelia between culture media were analyzed by $t$-test of five individual experiments.

Measurement of fungal growth The growth of P.expansum is expressed as a dry weight. P. expansum cultures in the apple juice media were filtered through No. 2 filter paper (Advantec Toyo Kaisha, Tokyo, Japan). Collected mycelia were frozen at $-80^{\circ} \mathrm{C}$, lyophilized and weighed.

Determination of patulin concentration in apple juice medium $\quad$ P. expansum culture in apple juice media was filtered through No. 2 filter paper. The filtrate was mixed with an equal volume of MeOH and then filtered through a 0.2- $\mu \mathrm{m}$ Ultrafree-MC Centrifugal Filter (Merck Millipore, Billerica, MA, USA). The mixture was subjected to high-performance liquid chromatography (HPLC) analysis with an HP-1100 highperformance liquid chromatograph equipped with a TSK gel ODS-100V separation column $(2 \times 150 \mathrm{~mm}, 3$ $\mu \mathrm{m}$; Tosoh, Tokyo, Japan). The HPLC system was connected to a UV detector and a mass selective detector with an electron spray ionization interface (Agilent Technologies, Santa Clara, CA, USA). The mobile phase A and B were Milli-Q water and MeCN, respectively. Separation was done at a flow rate of $0.2 \mathrm{~mL} / \mathrm{min}$ by the following linear gradient program: from 0.0 to $5.0 \mathrm{~min}, 95 \% \mathrm{~A}, 5 \% \mathrm{~B}$; from 5.0 to $13.0 \mathrm{~min}, 95 \% \mathrm{~A}, 5 \%$ B to $0 \% \mathrm{~A}, 100 \% \mathrm{~B}$; from 13.0 to $15.0 \mathrm{~min}, 0 \% \mathrm{~A}, 100 \% \mathrm{~B}$; from 15.0 to $15.1 \mathrm{~min}, 0 \% \mathrm{~A}, 100 \% \mathrm{~B}$ to $95 \% \mathrm{~A}$, $5 \% \mathrm{~B}$; and from 15.1 to $25.0 \mathrm{~min}, 95 \% \mathrm{~B}, 5 \% \mathrm{~A}$. The column temperature was $40^{\circ} \mathrm{C}$ and the injection volume was $2.0 \mu \mathrm{L}$. The UV absorbance at $290 \mathrm{~nm}$ and mass spectrometry at $\mathrm{m} / \mathrm{z} 153.0$ (negative, single-ion monitoring mode) were used in quantitative and qualitative analyses, respectively. The capillary voltage, fragmentor voltage, and temperature for mass spectrometry were $-6,000 \mathrm{~V}, 60 \mathrm{~V}$, and $340^{\circ} \mathrm{C}$, respectively. For the calibration standards, patulin standard solutions $(0,1,4,7,10,40,70$, and $100 \mu \mathrm{g} / \mathrm{mL})$ were prepared using 5\% MeCN. The measured patulin concentration was expressed as the concentration in the apple juice medium. 
Analysis of volatile compounds in apple juices by GC-MS coupled with headspace SPME Ten-milliliter aliquots of $1 \%$ apple juice in saturated $\mathrm{NaCl}$ solution were poured into 20 -mL glass vials, and then $50 \mu \mathrm{L}$ of 1 $\mathrm{mol} / \mathrm{L} \mathrm{HCl}$ was added. After the addition of $10 \mu \mathrm{L}$ of a mixture of methyl isobutyl ketone $(10 \mu \mathrm{g} / \mathrm{mL})$ and cyclooctyl alcohol $(10 \mu \mathrm{g} / \mathrm{mL})$ in EtOH as internal standards, the glass vials were sealed with PTFE-lined caps (GL Sciences Inc., Tokyo, Japan). Volatile compounds in headspace from the apple juices were extracted using a solid phase micro-extraction (SPME) fiber: 50 / $30 \mu \mathrm{m}$ DVB/CAR/PDMS, 2-cm length (SigmaAldrich, St. Louis, MO, USA). Extraction was performed at $60^{\circ} \mathrm{C}$ for 40 min under agitation (speed $400 \mathrm{rpm}$ ). After extraction, the SPME fiber was immediately inserted into the gas chromatography (GC) injector for thermal desorption for $5 \mathrm{~min}$ at $250^{\circ} \mathrm{C}$. An Agilent $6890 \mathrm{~N}$ gas chromatography system coupled to a 5975 inert Mass Selective Detector (MSD; Agilent Technologies) was used for capillary GC-mass spectrometry (MS) analyses in the electron impact mode. Volatile compounds were separated using a poly (ethylene glycol) column DB-WAX (Agilent Technologies, $30 \mathrm{~m} \times 0.25 \mathrm{~mm}$ i.d., film thickness $0.25 \mu \mathrm{m}$ ). The chromatographic conditions were as follows: injection system, splitless; injector temperature, $250^{\circ} \mathrm{C}$; temperature program, from 0 to $5 \mathrm{~min}$ at $40^{\circ} \mathrm{C}$, from 5 to $19 \mathrm{~min}$ at $15^{\circ} \mathrm{C} / \mathrm{min}$ and from 19 to $29 \mathrm{~min}$ at $250^{\circ} \mathrm{C}$; carrier gas, He; flow rate, $1.0 \mathrm{~mL} / \mathrm{min}$; transfer line to $\mathrm{MSD}, 250^{\circ} \mathrm{C}$. Estimation of compounds was made by the comparison of the mass spectra with those in the National Institute of Standards and Technology (NIST) mass spectra libraries (NIST, Gaithersburg, MD, USA). Linear Kovats indices of authentic compounds were also used to confirm the identification.

Effects of volatile compounds in apple juice on the patulin production by $\mathbf{P}$. expansum $\quad$ The 13 compounds of which concentrations were largely decreased by evaporation were added separately to RAJM $\mathrm{C}$ at the same concentrations as those determined for AJM C (original concentration), and also at the concentrations ten times higher than those in AJM C (ten-times concentration). AJM C was used as the positive control and RAJM C as the negative control. Volatile compound was added as an EtOH solution to the medium immediately before inoculation, the final concentration of $\mathrm{EtOH}$ in the medium was $0.1 \%$. The same amount of EtOH was added to positive and negative controls. P. expansum spores were inocultaed to apple juice media with the final spore concentration $1 \times 10^{4}$ spores $/ \mathrm{mL}$ and cultured statically at $25^{\circ} \mathrm{C}$ for $7 \mathrm{~d}$. The differences in the patulin concentrations between culture media were analyzed by $t$-test of five individual experiments.

Effect of 2-methylbutanoic acid on fungal growth and patulin production An EtOH solution of 2-methylbutanoic acid was added to RAJMs A-F at the same concentration as that in AJM C, and at the concentration ten-times higher than that in AJM C. RAJMs A-F without adding of 2-methylbutanoic acid were used as controls. A spore suspension of P. expansum was inoculated and cultured statically at $25^{\circ} \mathrm{C}$. After culturing for $7 \mathrm{~d}$, the dry weight of mycelia and the patulin concentration were determined. The differences in the weight of mycelia and the patulin concentrations between culture media were analyzed by $t$-test of five individual experiments. 


\section{Results}

Patulin production by $\mathbf{P}$. expansum in six media made from different commercial apple juices and the effect of evaporation of apple juices on patulin production A spore suspension of $P$. expansum was inoculated into the apple juice media (AJMs) A-F and the reconstructed apple juice media (RAJMs) A-F, and cultured at $25^{\circ} \mathrm{C}$ for $7 \mathrm{~d}$. The dry weight of mycelia was measured as an indicator of fungal growth, and the patulin amount in the culture medium was determined by LC/MS.

Table 1 shows the patulin production by $P$. expansum in the AJMs A-F of five individual experiments. The difference in apple juices markedly affected the patulin production by $P$. expansum. The lowest patulin concentration was $22.9 \pm 1.0 \mu \mathrm{g} / \mathrm{mL}$ in AJM E, and the highest patulin concentration was $167.3 \pm 24.3 \mu \mathrm{g} /$ $\mathrm{mL}$ in AJM C. Table 1 also shows the effect of the evaporation of apple juices on the patulin production by $P$. expansum in apple juice medium. The evaporation significantly reduced the patulin concentrations, by $43 \%$, $61 \%, 46 \%$ and $31 \%$ in apple juices A, B, C, and E, respectively (significance level 1\%). In apple juices D and $\mathrm{F}$, the evaporation scarcely changed the patulin concentrations in culture. These results suggested that some volatile compounds in apple juices might stimulate the patulin production by P. expansum.

Table 2 shows the fungal growth on the six AJMs of five individual experiments. The smallest dry weight of mycelia was recorded in AJM D (81\% of the average value in the six media), and the largest dry weight was obtained in AJM C (119\% of the average value), indicating that fungal growth was not greatly affected by the difference in apple juices. Table 2 also shows the fungal growth on the RAJMs A-F. Except for apple juice $\mathrm{E}$, the evaporation significantly increased the dry weight of mycelia. In particular, in apple juices $\mathrm{B}, \mathrm{C}$, and $\mathrm{D}$, the evaporation increased the dry weight of mycelia by 2.9-, 2.0-, and 2.3-fold, respectively. These results suggested that some volatile compounds in apple juices inhibited P. expansum growth.

Table 1. Patulin production in apple juice media and reconstructed apple juice media prepared from 6 apple juices

\begin{tabular}{ccc}
\hline & \multicolumn{2}{c}{ Patulin concentrations $(\mu \mathrm{g} / \mathrm{mL})$} \\
\cline { 2 - 3 } Apple juice & AJM $^{\mathrm{a}}$ & \multicolumn{1}{c}{ RAJM $^{\mathrm{b}}$} \\
\cline { 2 - 3 } & mean $\pm \mathrm{SD}^{\mathrm{c}}$ & mean $\pm \mathrm{SD}^{\mathrm{c}, \mathrm{d}}$ \\
\hline A & $55.4 \pm 7.8$ & $31.6 \pm 10.9^{* *}$ \\
B & $27.9 \pm 7.4$ & $11.0 \pm 2.4^{* *}$ \\
C & $167.3 \pm 24.3$ & $90.3 \pm 33.0^{* *}$ \\
D & $31.2 \pm 3.8$ & $29.8 \pm 3.3$ \\
E & $22.9 \pm 1.0$ & $15.7 \pm 1.3^{* *}$ \\
F & $39.3 \pm 12.1$ & $25.7 \pm 3.5$ \\
\hline
\end{tabular}

\footnotetext{
${ }^{\mathrm{a}} \mathrm{AJM}$ : apple juice medium without evaporation.

${ }^{\mathrm{b}} \mathrm{RAJM}$ : reconstructed apple juice medium.

${ }^{\mathrm{c}}$ The means and SD for five individual experiments.

${ }^{\mathrm{d}}$ Asterisks indicate significant differences $\left({ }^{* *} p<0.01\right)$ from AJMs values as determined by Aspin-Welch's $t$-test.
} 
Table 2. Fungal growth in apple juice media and reconstructed apple juice media prepared from 6 apple juices

\begin{tabular}{ccc}
\hline & \multicolumn{2}{c}{ Dry weights of mycelia (mg) } \\
\cline { 2 - 3 } Apple juice & \multicolumn{1}{c}{ AJM $^{\mathrm{a}}$} & RAJM $^{\mathrm{b}}$ \\
\cline { 2 - 3 } & mean $\pm \mathrm{SD}^{\mathrm{c}}$ & mean $\pm \mathrm{SD}^{\mathrm{c}, \mathrm{d}}$ \\
\hline A & $56.6 \pm 16.0$ & $82.9 \pm 19.5^{*}$ \\
$\mathrm{~B}$ & $51.1 \pm 11.4$ & $146.1 \pm 17.0^{* *}$ \\
$\mathrm{C}$ & $65.3 \pm 7.8$ & $129.2 \pm 22.7^{* *}$ \\
$\mathrm{D}$ & $44.6 \pm 4.0$ & $101.0 \pm 11.9^{* *}$ \\
$\mathrm{E}$ & $57.5 \pm 17.0$ & $56.8 \pm 7.2$ \\
$\mathrm{~F}$ & $54.1 \pm 5.2$ & $83.8 \pm 21.9^{*}$ \\
\hline
\end{tabular}

\footnotetext{
${ }^{\mathrm{a}} \mathrm{AJM}$ : apple juice medium without evaporation.

${ }^{\mathrm{b}} \mathrm{RAJM}$ : reconstructed apple juice medium.

${ }^{\mathrm{c}}$ The means and SD for five individual experiments.

${ }^{\mathrm{d}}$ Asterisks indicate significant differences $\left({ }^{*} p<0.05,{ }^{* *} p<0.01\right)$ from AJMs

values as determined by Aspin-Welch's $t$-test.
}

\section{Analysis of volatile compounds in original and evaporated apple juices Since the volatile compounds in} the apple juice might affect the growth of $P$. expansum and its patulin production, the contents of volatile compounds in the apple juices A-F and in the reconstructed apple juices A-F were determined before sterilization. The volatile compounds of these 12 apple juices were analyzed by GC/MS coupled with headspaceSPME, and a number of volatile compounds were detected as shown in Supplementary Table 1.

From detected compounds in 12 apple juices by GC/MS analysis, 13 compounds were selected on the basis of two criteria. First, we selected the compounds of which intensity values were larger than one-tenth of those of internal standards in the original apple juice C. The greatest amount of patulin was produced in AJM $\mathrm{C}$ among the six AJMs. Second, we selected the compounds of which the concentrations were reduced to one-fifth or less by evaporation. Those 13 compounds were identified to be 2 -methylpropyl acetate, ethyl butyrate, ethyl 2-methylbutanoate, butyl acetate, hexanal, 2-methylbutyl acetate, 1-butanol, (E)-2-hexenal, 3-methyl-1-butanol, hexyl acetate, octanal, 1-hexanol, and 2-methylbutanoic acid by comparison of retention time on GC and MS fragmentation pattern with authentic standard compounds. Their actual concentrations in the original apple juices and reconstructed apple juices were determined more accurately by standard curves generated for each compound, and are shown in Table 3. Indeed, the selected compounds were confirmed to be present at a high concentration in original juice $\mathrm{C}$, and their concentrations were greatly reduced by evaporation. 


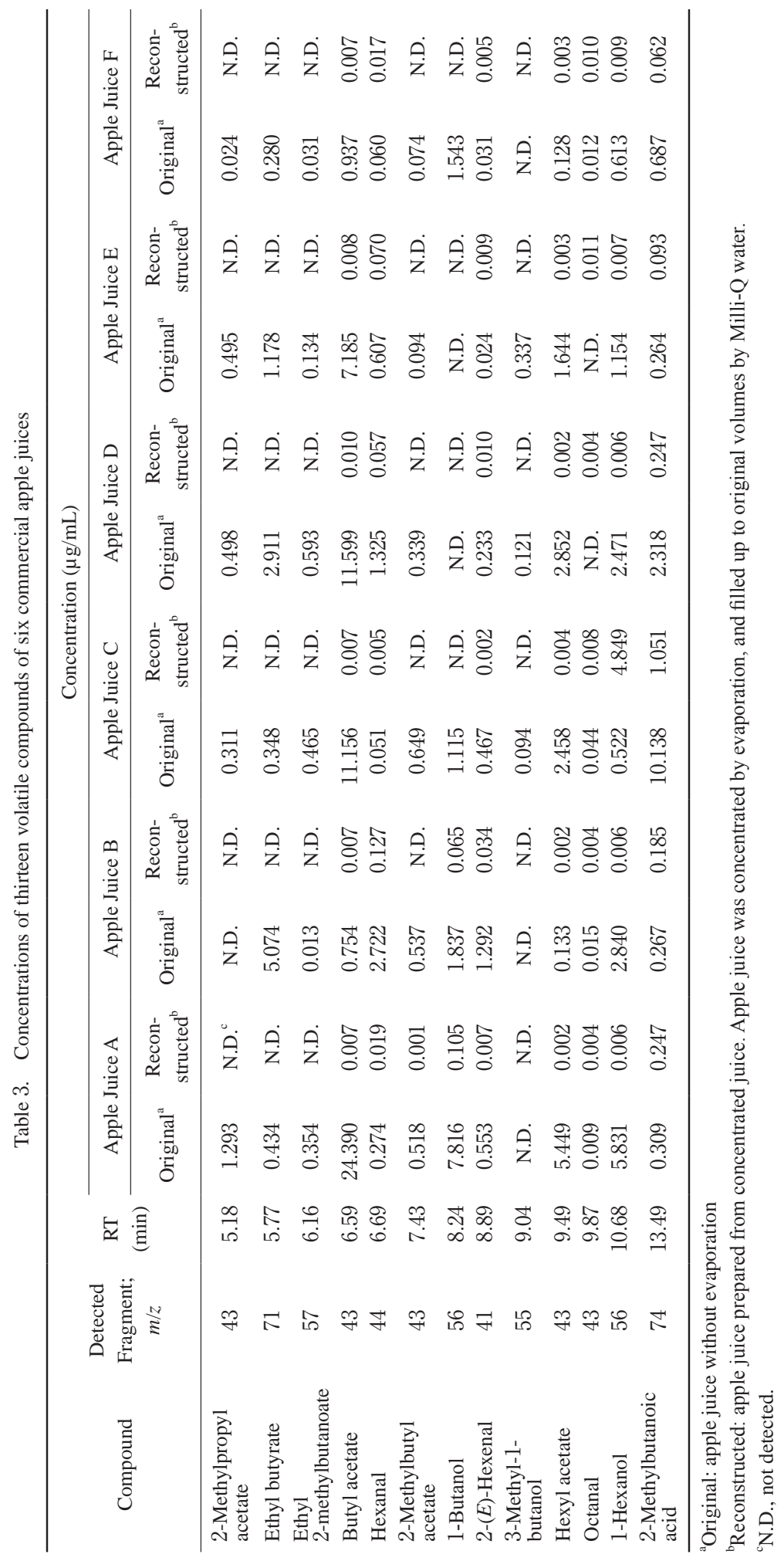



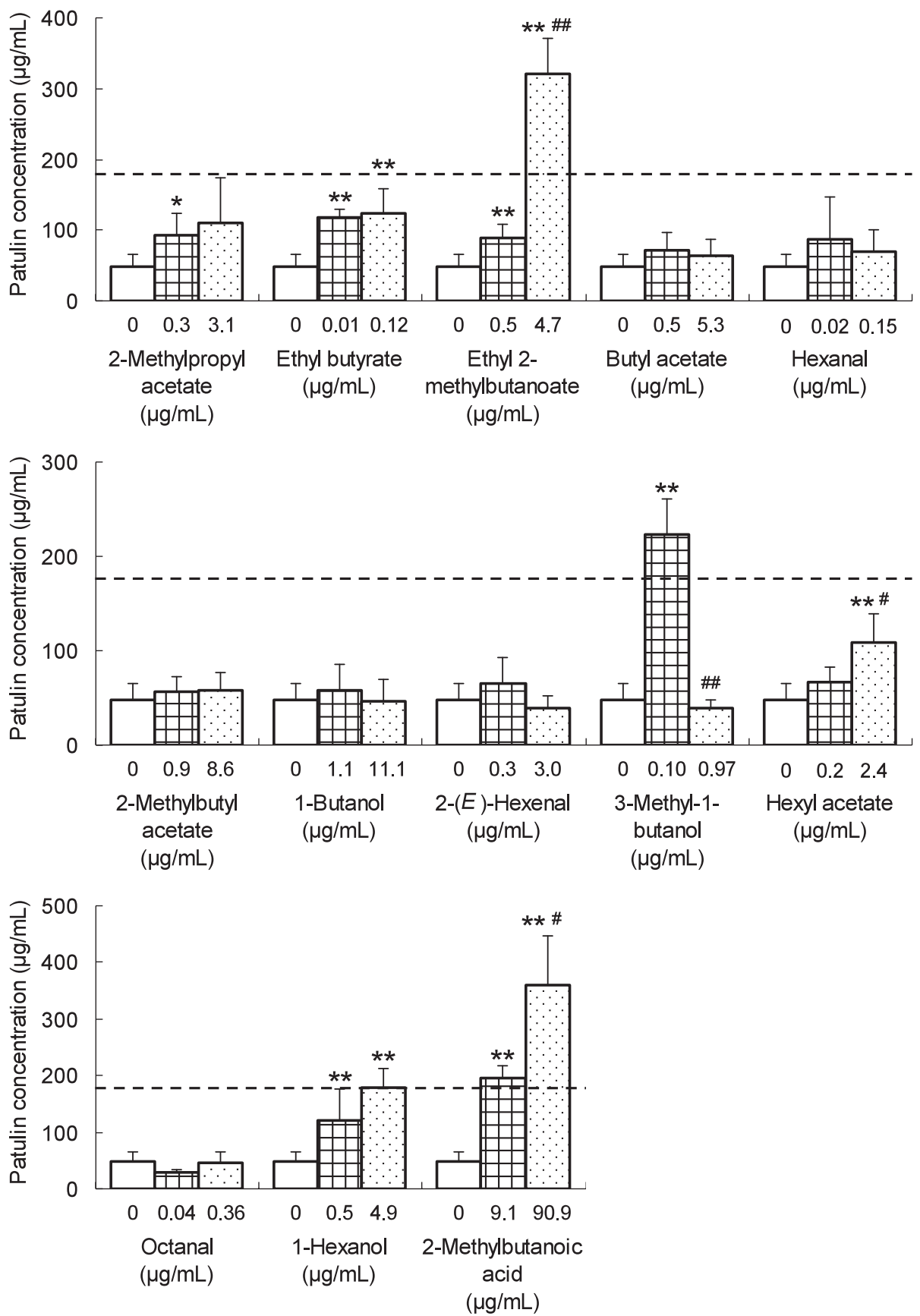

Fig. 1. Effects of the 13 volatile compounds on the patulin production by $P$. expansum in apple juice medium. The 13 compounds were added to RAJM C. The final concentrations of compounds were adjusted to the same concentrations as AJM C (center bars), and ten times concentrations (right bars). AJM C (dotted line) and RAJM C (left bars) were used as positive and negative controls. The patulin concentrations in the graph are expressed as the means $\pm \mathrm{SD}$ of five individual experiments. $* p<0.05$, $* * p<0.01$ compared with values for negative controls; ${ }^{\#} p<0.05,{ }^{\# \#} p<0.01$ compared with values for original concentrations. 
Effects of volatile compounds in apple juice on the patulin production by P. expansum The effects of the above-mentioned 13 volatile compounds on the patulin production by P. expansum were investigated using RAJM C. The 13 compounds were added separately to RAJM C at the same concentrations as those determined for AJM C shown in Table 2 (original concentration), and also at the concentrations ten times higher than those in AJM C (ten-times concentration). AJM C and RAJM C were used as positive and negative controls. The patulin concentrations in culture media were determined after culturing for $7 \mathrm{~d}$.

Figure 1 demonstrates that the addition of butyl acetate, hexanal, 2-methylbutyl acetate, 1-butanol, (E)-2-hexenal, and octanal did not affect the patulin production. By contrast, 2-methylpropyl acetate, ethyl butyrate, ethyl 2-methylbutanoate, hexyl acetate, 1-hexanol, and 2-methylbutanoic acid increased the patulin concentration in the cultures. In the media to which 2-methylpropyl acetate or ethyl butyrate were added up to original concentrations, the patulin amounts in the cultures were significantly increased compared to that in negative control. However, when two compounds were added up to ten-times concentrations, the patulin concentration in cultures did not increase any further. The addition of 3-methyl-1-butanol and 2-methylbutanoic acid up to the original concentrations stimulated the patulin production up to or over the patulin concentration $(175.2 \pm 71.5 \mu \mathrm{g} / \mathrm{mL})$ in the positive control, AJM C. Also, the addition of ethyl 2-methylbutanoate, 1-hexanol, and 2-methylbutanoic acid up to the ten-times concentrations stimulated the patulin production up to or over the patulin level in the positive control, whereas the addition of 3-methyl-1-butanol up to the ten-times concentration did not affect it.

Effect of 2-methylbutanoic acid on fungal growth and patulin production Since 2-methylbutanoic acid effectively stimulated the patulin production in RAJM C (Fig. 1), its effect on the patulin production by $P$. expansum were investigated in the other five apple juices. 2-Methylbutanoic acid was added to RAJMs A-F at the same concentration $(9.1 \mu \mathrm{g} / \mathrm{mL})$ as that in AJM C, and at the concentration $(90.9 \mu \mathrm{g} / \mathrm{mL})$ ten times higher than that in AJM C. The six RAJMs were used as negative controls. The patulin concentration (Fig. $2 \mathrm{~A}$ ) and fungal growth (Fig. 2B) in culture media were determined after $7 \mathrm{~d}$ culturing.

In the cultures of RAJMs A, B, C, D and E, the addition of 2-methylbutanoic acid significantly increased the patulin amount in a concentration-dependent manner, and in RAJM F, a weak tendency to increase patulin concentration was observed. Conversely, in the A-F media, the addition of 2-methylbutanoic acid significantly decreased the dry weights of mycelia of $P$. expansum. The relationship between decrease in the fungal growth and enhancement of patulin production in apple juice medium by the addition of 2-methylbutanoic acid was the same as the relationship observed for some AJMs and RAJMs. That is, the evaporation significantly reduced the patulin productions and significantly increased the fungal growth in apple juices $\mathrm{A}, \mathrm{B}$, and C (Table 1, 2).

Thus the relation between concentrations of 2-methylbutanoic acid (Table 3) and patulin production (Table 1) in the six AJMs and six RAJMs was investigated; a positive correlation was observed between the 2 -methylbutanoic acid concentration and the patulin production in twelve apple juice media as shown in Figure 3.

\section{Discussion}

The present study demonstrates that patulin production by $P$. expansum depends on media made from the 
A

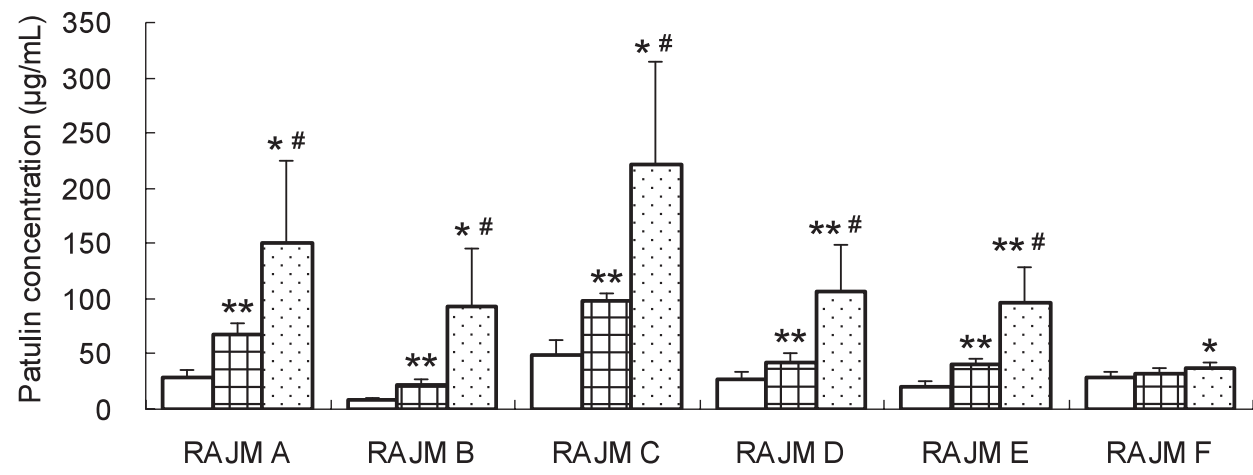

B

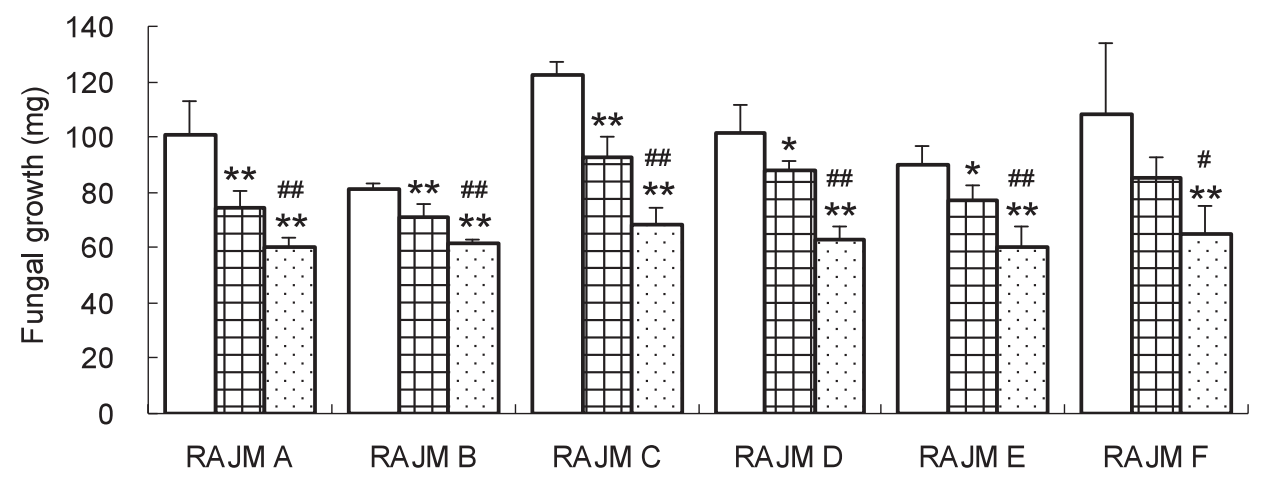

Fig. 2. Effects of 2-methylbutanoic acid on patulin production (A) and fungal growth (B) in six apple juice media. 2-Methylbutanoic acid was added to RAJMs A-F. The final concentration were adjusted to the same concentration as AJM C $(9.1 \mu \mathrm{g} / \mathrm{mL}$ : center bars), and the ten times concentration ( $90.9 \mu \mathrm{g} / \mathrm{mL}$ : right bars). RAJMs A-F ( $0 \mu \mathrm{g} / \mathrm{mL}$ : left bars) were used as negative controls. The patulin concentration and fungal growth in the graph are expressed as the means \pm SD of five individual experiments. ${ }^{*} p<0.05, * * p<0.01$ compared with values for negative controls; ${ }^{*} p<0.05,{ }^{\#} p<0.01$ compared with values for original concentrations.

six different apple juices. Evaporation of these juices reduced the patulin production and simultaneously increased fungal growth, suggesting that some volatile componds in these juices stimulate patulin production and inhibit fungal growth. In fact, seven volatile compounds ( 2-methylpropyl acetate, ethyl butyrate, ethyl 2-methylbutanoate, 3-methyl-1-butanol, hexyl acetate, 1-hexanol, and 2-methylbutanoic acid) in the apple juices were found to enhance the patulin production.

Especialy, the addition of 2-methylbutanoic acid stimulated patulin production in the six different apple juices in a concentration-dependent manner. We also observed a positive correlation between the 2-methylbutanoic acid concentration and the patulin production in the six AJMs and six RAJMs. The concentration of 2-methylbutanoic acid may affect the patulin production of P. expansum in apple juice. However, the data for AJM D largely departed from this correlation; even though the concentration of 2-methylbutanoic acid was $2.32 \mu \mathrm{g} / \mathrm{mL}$ (Table 3), the patulin concentration was $31.2 \pm 3.8 \mu \mathrm{g} / \mathrm{mL}$ (Table 1). More experiments are needed before determining completely the role of 2 -methylbutanoic acid on patulin production of $P$. expansum 


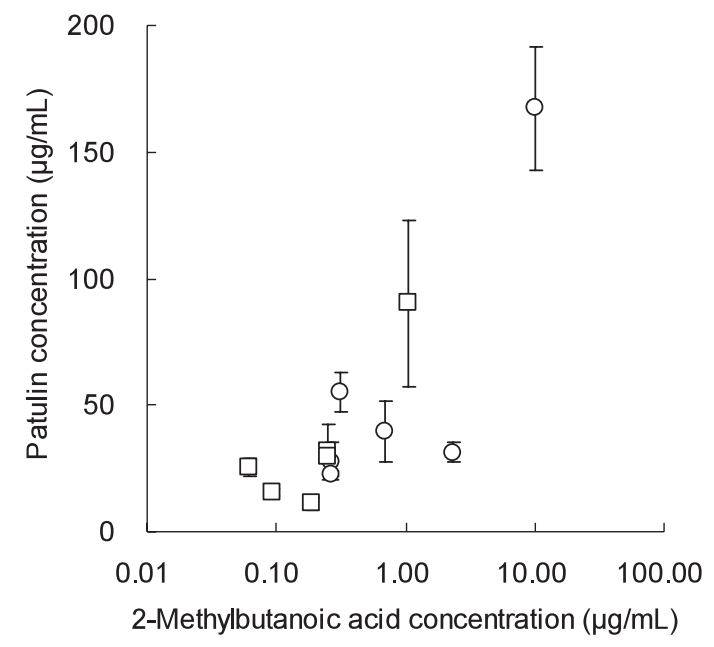

Fig. 3. The relationship between the 2 -methylbutanoic acid concentration and the patulin concentration in the apple juice media. The concentrations of 2 -methylbutanoic acid and patulin were determined after incubating P. expansum in six AJMs ( $\bigcirc$ ) and six RAJMs ( $\square$ ) at $25^{\circ}$ $\mathrm{C}$ for $7 \mathrm{~d}$. Symbols and error bars represent the means $\pm \mathrm{SD}$ of five individual experiments.

in apple juice.

The addition of ethyl 2-methylbutanoate also stimulated the patulin production in RAJM C at a lower concentration than that of 2-methylbutanoic acid, indicating that ethyl 2 -methylbutanoate is a more effective compound on patulin production than 2-methylbutanoic acid. This may imply that the stimulating activity of these volatile compounds is affected by their hydrophobicity and permeability. The 2-methylbutanoyl group might be an essential structural unit to elicit the enhancement of patulin production of $P$. expansum, and in that sense, it is of interest to analyze the structure-activity relationship among 2-methylbutanoic acid derivatives.

Our previous study showed that some aliphatic aldehydes, such as octanal, increased the patulin production of $P$. expansum in apple juice without affecting fungal growth ${ }^{25}$. Although the most effective concentration of octanal on patulin production in our previous study was $300 \mu \mathrm{g} / \mathrm{mL}$, the maximum concentration of octanal in the six apple juices used in the present study was only $0.04 \mu \mathrm{g} / \mathrm{mL}$, in AJM C. Therefore, aliphatic aldehydes at their actual concentrations in apple juice can not stimulate the patulin production of $P$. expansum. In contrast, 2-methylbutanoic acid and its ethyl ester stimulated the patulin production by $P$. expansum at their actual concentrations in apple juice.

Manganese was reported to play an important role in patulin production by P. expansum ${ }^{28}$. We therefore suspected that there were significant differences among the juices in the contents of metals, and determined the contents of metals in the six apple juices. Potassium, phosphorous, sodium, magnesium, calcium, and manganese were detected in all six juices, but there was no correlation between the patulin production and contents of any of these metals (data not shown).

The present study showed that at least five other compounds, besides 2-methylbutanoic acid and its ethyl ester, stimulated patulin production. In apple juice, they may interact with each other in the stimulation of patulin production. The identification of such interactions in the patulin production of $P$. expansum will 
contribute to the development of methods to control the patulin contamination of apples and apple products.

Ethyl 2-methylbutanoic acid is present in many types of fruit, and descriptions of its aroma include "fruity" and "sweet" aroma notes; it apparently contributes greatly to the aroma of many fruits, including apple ${ }^{29-33}$. By contrast, the odor of 2-methylbutanoic acid is described as "cheesy" or "rancid," and it is very different from apples' aroma ${ }^{30,34)}$. The results of these sensory evaluations indicate that 2-methylbutanoic acid is not necessarily an important aromatic component in apples and apple juice. Several maize lines are known to have resistance to A. flavus infection and aflatoxin contamination, and the elucidation of their mechanism has been carried out by molecular breeding ${ }^{35}$. Our present results demonstrate that the selective breeding of an apple cultivar is a promising approach for reducing the patulin contamination without the loss of sensory values if the cultivar does not contain compounds that stimulate the patulin production of $P$. expansum and simultaneously have little effect on apple aroma (such as 2-methylbutanoic acid). Both a reduction of the risk of health hazards and a reduction of economic losses could thus be achieved by this approach.

\section{References}

1 ) Birkinshaw, J.H., Bracken, A., Michael, S.E., Raistrick, H.: Patulin in the common cold collaborative research on a derivative of Penicillium patulum Bainier: II. Biochemistry and Chemistry. Lancet, 242, 625-630 (1943)

2 ) Frisvad, J.C.: Physiological criteria and mycotoxin production as AIDS in identification of common asymmetric penicillia. Appl Environ Microbiol, 41, 568-579 (1981)

3 ) Paucod, J.C., Krivobok, S., Vidal, D.: Immunotoxicity testing of mycotoxins T-2 and patulin on Balb/c mice. Acta Microbiologica Hungarica, 37, 331-339 (1990)

4 ) Devaraj, H., Radha Shanmugasundaram, K., Shanmugasundaram, E.R.: Neurotoxic effect of patulin. Indian J Exp Biol, 20, 230-231 (1982)

5 ) U.S.Food and Drug Administration Compliance policy guid.: Compliance Policy Guidance for FDA Staff. Sec. 510.150. Apple juice, apple juice concentrates, and apple juice products - Adulteration with Patulin. (2004)

6 ) European Commission. Commission Regulation (EC) No 1881/2006 of 19 December 2006 setting maximum levels for certain contaminants in foodstuffs. Off J Eur Union, L364, 5-24 (2006)

7 ) Ministry of Health, Labour and Welfare of Japan: Notification No. 369 (2003)

8 ) Cappellini, R.A., Ceponis, M.J., Lightner, G.W.: Disorders in apple and pear shipments to the New York market, 1972-1984. Plant Disease, 71, 852-856 (1987)

9 ) Kabak, B., Dobson, A.D., Var, I.: Strategies to prevent mycotoxin contamination of food and animal feed: a review. Crit Rev Food Sci Nutr, 46, 593-619 (2006)

10) Salomão, B.C., Aragão, G.M., Churey, J.J., Padilla-Zakour, O.I., Worobo, R.W.: Influence of storage temperature and apple variety on patulin production by Penicillium expansum. J Food Prot, 72 , 1030-1036 (2009)

11) Morales, H., Marín, S., Rovira, A., Ramos, A.J., Sanchis, V.: Patulin accumulation in apples by Penicillium expansum during postharvest stages. Lett Appl Microbiol, 44, 30-35 (2007)

12) Moodley, R.S., Govinden, R., Odhav, B.: The effect of modified atmospheres and packaging on patulin production in apples. J Food Prot, 65, 867-871 (2002) 
13) Baert, K., Devlieghere, F., Flyps, H., Oosterlinck, M., Ahmed, M.M., Rajković, A., Verlinden, B., Nicolaï, B., Debevere, J., de Meulenaer, B.: Influence of storage conditions of apples on growth and patulin production by Penicillium expansum. Int J Food Microbiol, 119, 170-181 (2007)

14) Lennox, J.E., McElroy, L.J.: Inhibition of growth and patulin synthesis in Penicillium expansum by potassium sorbate and sodium propionate in culture. Appl Environ Microbiol, 48, 1031-1033 (1984)

15) Dong, Q., Manns, D.C., Feng, G., Yue, T., Churey, J.J., Worobo, R.W.: Reduction of patulin in apple cider by UV radiation. J Food Prot, 73, 69-74 (2010)

16) Arroyo, T.F., Moreno, J., Daza, P., Boianova, L., Romero, F.: Antifungal activity of strawberry fruit volatile compounds against Colletotrichum acutatum. J Agric Food Chem, 55, 5701-5707 (2007)

17) Utama, I.M.S., Willis, R.B.H., Ben-yehoshua, S., Kuek, C.: In vitro efficacy of plant volatiles for inhibiting the growth of fruit and vegetable decay microorganisms. J Agric Food Chem, 50, 6371-6377 (2002)

18) Cleveland, T.E., Carter-Wientjes, C.H., DeLucca, A.J., Boue, S.M.: Effect of soybean volatile compounds on Aspergillus flavus growth and aflatoxin production. J Food Sci, 74, H83-H87 (2009)

19) Vaughn, S.F., Spencer, G.F., Shasha, B.S.: Volatile compounds from raspberry and strawberry fruit inhibit postharvest decay fungi. J Food Sci, 58, 793-796 (1993)

20) Archbold, D.D., Hamilton-Kemp, T.R., Barth, M.M., Langlois, B.E.: Identifying natural volatile compounds that control gray mold (Botrytis cinerea) during postharvest storage of strawberry, blackberry, and grape. J Agric Food Chem, 45, 4032-4037 (1997)

21) Nakamura, S., Hatanaka, A.: Green-leaf-derived C6-aroma compounds with potent antibacterial action that act on both Gram-negative and Gram-positive bacteria. J Agric Food Chem, 50, 7639-7644 (2002)

22) Kubo, I., Fujita, K., Kubo, A., Nihei, K., Lunde, C.S.: Modes of antifungal action of (2E)-alkenals against Saccharomyces cerevisiae. J Agric Food Chem, 51, 3951-3957 (2003)

23) Neri, F., Mari, M., Brigati, S.: Control of Penicillium expansum by plant volatile compounds. Plant Pathol, 55, 100-105 (2006)

24) Neri, F., Mari, M., Menniti, A.M., Brigati, S.: Activity of trans-2-hexenal against Penicillium expansum in 'Conference’ pears. J Appl Microbiol, 100, 1186-1193 (2006)

25) Taguchi, T., Kozutsumi, D., Nakamura, R., Sato, Y., Ishihara, A., Nakajima, H.: Effects of aliphatic aldehydes on the growth and patulin production of Penicillium expansum in apple juice. Biosci Biotechnol Biochem, 77, 138-144 (2013)

26) Zeringue, H.J. Jr.: Effect of $\mathrm{C} 6$ to $\mathrm{C} 9$ alkenals on aflatoxin production in corn, cottonseed, and peanuts. Appl Environ Microbiol, 57, 2433-2434 (1991)

27) Karlshøj, K., Nielsen, P.V., Larsen, T.O.: Prediction of Penicillium expansum spoilage and patulin concentration in apples used for apple juice production by electronic nose analysis. J Agric Food Chem, 55, 4289-4298 (2007)

28) Dombrink-Kurtzman, M.A., Blackburn, J.A.: Evaluation of several culture media for production of patulin by Penicillium species. Int J Food Microbiol, 98, 241-248 (2005)

29) Sampaio, K.L., Garruti, D.S., Franco, M.R., Janzantti, N.S., Da Silva, M.A.: Aroma volatiles recovered in the water phase of cashew apple (Anacardium occidentale L.) juice during concentration. J Sci Food Agric, 91, 1801-1809 (2011)

30) Du, X., Plotto, A., Baldwin, E., Rouseff, R.: Evaluation of volatiles from two subtropical strawberry cultivars using GC-olfactometry, GC-MS odor activity values, and sensory analysis. J Agric Food Chem, 
59, 12569-12577 (2011)

31) Montero-Calderón, M., Rojas-Graü, M.A., Martín-Belloso, O.: Aroma profile and volatiles odor activity along gold cultivar pineapple flesh. J Food Sci, 75, S506-512 (2010)

32) Miyazaki, T., Plotto, A., Baldwin, E.A., Reyes-De-Corcuera, J.I., Gmitter, F.G. Jr.: Aroma characterization of tangerine hybrids by gas-chromatography-olfactometry and sensory evaluation. J Sci Food Agric, 92, 727-735 (2012)

33) Kraujalytè, V., Leitner, E., Venskutonis, P.R.: Characterization of Aronia melanocarpa volatiles by headspace-solid-phase microextraction (HS-SPME), simultaneous distillation/extraction (SDE), and gas chromatography-olfactometry (GC-O) methods. J Agric Food Chem, 61, 4728-4736 (2013)

34) Xu, Y., Fan, W., Qian, M.C.: Characterization of aroma compounds in apple cider using solvent-assisted flavor evaporation and headspace solid-phase microextraction. J Agric Food Chem, 55, 3051-3057 (2007)

35) Cray, J.W., Rajasekaran, K., Brown, R., Luo, M., Chen, Z.U., Bhatnagar, D.: Developing resistance to aflatoxin in maize and cottonseed. Toxins, 3, 678-696 (2011)

\section{Supplementary materials}

Supplementary materials may be found in the online version of this article:

Supplementary Table 1. Relative peak area of volatile compounds from six apple juices determined by GC/MS analysis coupled with headspace SPME. 
Supplementary Table 1. Relative peak area of volatile compounds from six apple juices determined by GC/MS analysis coupled with headspace SPME

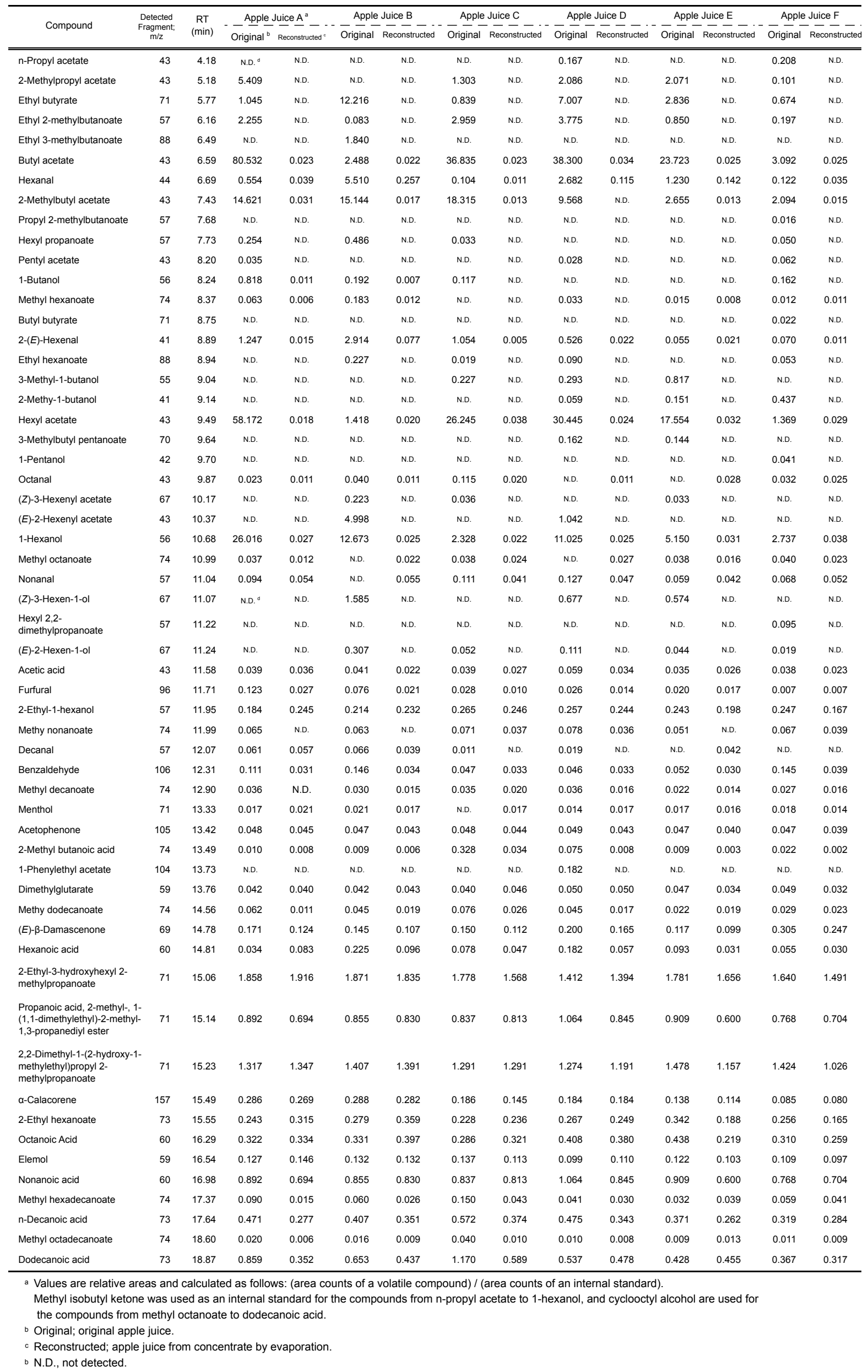

